\title{
APPLICATION OF SHAPE-BASED SIMILARITY QUERY FOR AERODYNAMIC OPTIMIZATION OF WIND TUNNEL PRIMARY NOZZLE
}

\begin{abstract}
Jan Koláŕ ${ }^{\bullet}$
Abstract: The aerodynamic shape optimization of the supersonic flat nozzle is the aim of proposed paper. The nozzle discussed, is applied as a primary nozzle of the inlet part of supersonic wind tunnel. Supersonic nozzles of the measure area inlet parts need to guarantee several requirements of flow properties and quality. Mach number and minimal differences between real and required velocity and turbulence profiles at the nozzle exit are the most important parameters to meet. The aerodynamic shape optimization of the flat $2 D$ nozzle in CFD is employed to reach as uniform exit velocity profile as possible, with the mean Mach number 1.4. Optimization process does not use any of standard routines of global or local optimum searching. Instead, newly formed routine, which exploits shape-based oriented sequence of nozzles, is used to research within whole discretized parametric space. The movement within optimization process is not driven by gradient or evolutionary too, instead, the Path of Minimal Shape Deformation is followed. Dynamic mesh approach is used to deform the shape and mesh from the actual nozzle to the subsequent one. Dynamic deformation of mesh allows to speed up whole converging process as an initialization of flow at the newly formed mesh is based on afore-computed shape. Shape-based similarity query in field of supersonic nozzles is discussed and applied. Evolutionary technique with genetic algorithm is used to search for minimal deformational path. As a result, the best variant from the set of solved shapes is analyzed at the base of momentum coefficient and desired Mach number at the nozzle exit.
\end{abstract}

\section{INTRODUCTION}

Experimental devices for basic research in field of supersonic or transonic speed needs to be designed very carefully to ensure flawless observations and measurements as shown by Shapiro in [1]. Primary nozzle of supersonic wind tunnel is one of those parts, whose quality typically determines properties of whole wind tunnel. Atmospheric air, sucked into measure area through the primary nozzle, is ideally shock-free at the measure area intake. Beyond this, it should provide flow field without large gradients of flow variables and with uniform velocity profile at the exit. Interaction of shock waves with boundary layer further affects flow as shown by Moeckel [2] and Kolář [3]. Boundary layer separation is inacceptable in design-regime and should be avoided or minimized in other regimes, thus. Operating regimes of supersonic nozzles were studied by Shapiro in [1]. Basically, the nozzle design should ensure required Mach number in measure area. In this paper, desired Mach number is established to $M a_{i d}=1.4$ at the base of ejector-induced under-pressure, available at the nozzle exit, computed by Dvořák in [4], [5].

\footnotetext{
- Kolár Jan, Department of Power Engineering, Technical University of Liberec, Studentská 2, Liberec 46117, Czech Republic, jan.kolar1@tul.cz
} 


\section{Methods}

The methodology of nozzle shape optimization is not based on any of the gradient or population strategy discoussed e.g. in [6]. Instead, set of vectors from whole parametric space defined by the discretization step of every single parameter is established. Steps of parameters are chosen in order to represent data from whole parametric space with acceptable density. The limitation of discretization step size results from limited memory capacities of actual work station. In the consequential manipulating with set of vectors within population of shapes, whole population needs to be handled as spatial data fields, where population $\mathrm{P}$ is declared as field of real numbers:

real $R$ [size of population][number of vectors][number of parameters].

In our task, defined by five independent parameters ( $\mathrm{P} 1, \mathrm{P} 2, \mathrm{P} 3, \mathrm{P} 4, \mathrm{P} 5)$ seen at the figure 1 and population size of fifty vector sequences, resuting memory capabilities enables three variations of every parameter as can be seen in table 1.

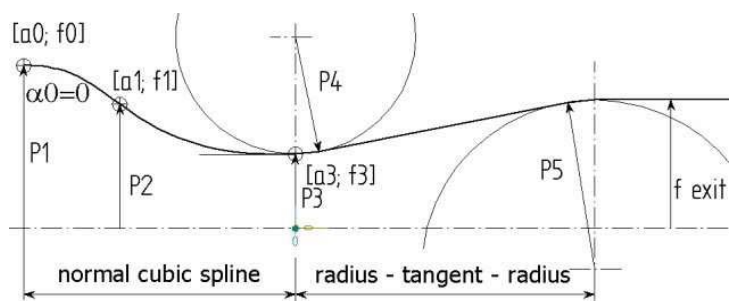

Figure 1: Scheme of optimization task and its parameterization.

\begin{tabular}{|c|c|c|c|}
\hline$P 1[\mathrm{~mm}]$ & 22 & 24 & 26 \\
\hline$P 2[\mathrm{~mm}]$ & 18 & 20 & 22 \\
\hline$P 3[\mathrm{~mm}]$ & 10.8 & 11.2 & 11.6 \\
\hline$P 4[\mathrm{~mm}]$ & 10 & 30 & 50 \\
\hline$P 5[\mathrm{~mm}]$ & 10 & 30 & 50 \\
\hline$a 0=-85 \mathrm{~mm}, \mathrm{a} 1=-40 \mathrm{~mm}$, aexit=26 mm, fexit=12.5mm \\
\hline \multicolumn{4}{|c|}{}
\end{tabular}

Table 1: Computed variants and other dimensions of task. $n=3^{5}=243$ variants.

Approximate nozzle shape, computed in [7], is composed from two separate parts $y 1_{(x)}$, $y 2_{(x)}$, whose are attached one in other in the point $x=0, y=f 3=P 3=y 1_{(0)}=y 2_{(0)}$, where also $y 1^{\prime}(0)=y 2^{\prime}(0)$. Shape of the converging part of the nozzle in the interval of $x \in(a 0, a 3)$ is defined by normal cubic spline. Gauss elimination is used to solve the system of linear algebraic equations, which describes spline as a smooth function of $x$-coordinate. This allows modification of converging part at the base of only three parameters. Shape of diverging part in the interval of $x \in\left(a 3, x_{\text {exit }}\right)$ is based on two circles with radii P4, P5 connected tangentially one in other. Solving of transitional points, where circular shape changes its character to linear are solved for every variation in separate, respectively. Further, presented routine does not evaluate objective for vectors in random order. Instead the path of minimal shape deformation, needed to deform the nozzle shape over all selected vectors

$V_{i}=\left(P 1_{i}, P 2_{i}, P 3_{i}, P 4_{i}, P 5_{i}\right)$, where for $\forall i$ is $V_{i} \in($ optimization space) and $i=0,1,2, \ldots$, SIZE where SIZE is the size of population,

is followed. Unfortunately, finding of minimal deformation path (MDP) is not from those of trivial tasks [8]. This task is identical to well known travelling salesman problem (TSP). Given a list of $K$ cities and their pairwise distances (deformation), the task is to find the 
shortest possible tour that visits each city exactly once. In this task the salesman has to visit cities of given locations, starting from his city and returning back. In the theory of computational complexity, the decision version of TSP belongs to the class of NPcomplete problems. The only approach able to improve objective are heuristic algorithms. One of the most efficient is the genetic algorithm (GA). Several modifications of standard approach is used to improve convergence to global optimum and help objective to improve.

\section{Genetic algorithm for solution of the minimal deformation path (MDP)}

Genetic algorithm (GA) was firstly introduced by J. Holland and may be studied in [8] and [9]. For TSP, many artificial modifications of evolutionary algorithm have been developed and tested. Most significant task about GA, is the methodology of coding the individuals. As the GA further works with individuals represented by its chromosomes and all operators are applied to theme, technique of individual coding has a significant impact to EA advance as mentioned by Hynek in [8] and Zelinka in [10]. If there is no suitable coding applied, even an infinitesimal change of chromosome code may have large impact to the objective function. Typical example is a chromosome handled in binary code, where even one binary-unite-change in number $(11010110)_{2}=214$ produces the $(10010110)_{2}=150$ - The GA than betrays itself. We decided to use parameter based code, where the chromosome gets value of parameters as a real number. Selection of individuals for further evolution is based on fitness proportionate selection with its modification: stochastic universal sampling. More detailed view can is taken by Hynek in [8]. At the figure 2, there are four balls to select the equal number of individuals (nozzle sequences). The individual is selected when the randomly generated number $r$ (equal to $\alpha$ at figure 2) is such, that $\bar{f}_{i-1}<r \leq \bar{f}_{i}$, SIZE is the size of population $f$ is the individual fitness and $\bar{f}_{i}$ is defined as follows

$$
\bar{f}_{i}=\sum_{j^{\prime}=1}^{i} \frac{f_{i}}{\sum_{k=1}^{S I Z E} f_{k}}, \text { where } i=1,2, \ldots, \text { SIZE } .
$$

One of the most effective recombination operators for TSP is the edge recombination crossover (ERX). In this method the only one child, is reproduced from parent's couple. Its chromosome path is combination of mothers and fathers chromosome part of path. See more details in [8].

\section{Definition of MDP by shape-based similarity query}

It is difficult to define the similarity of shapes or trajectory in a space. Most of database systems adopt the Euclidean distance of two data sequences for analysis, where each has $\mathrm{n}$ values. Similarity is given by the Euclidean distance between vectors in $\mathbf{R}^{\mathbf{2}}$. In Yanagisawa [11], there are two data series $c=\left\langle w_{1}, w_{2}, \ldots, w_{n}\right\rangle, c^{\prime}=\left\langle w_{1}^{\prime}, w_{2}^{\prime}, \ldots, w_{n}^{\prime}\right\rangle$.Than, distance $D\left(c, c^{\prime}\right)$ is defined as follows

$$
D\left(c, c^{\prime}\right)=\sqrt{\left(w_{1}-w_{1}^{\prime}\right)^{2}+\cdots\left(w_{n}-w_{n}^{\prime}\right)^{2}} \text {. }
$$

Based on this definition, we consider the spatial shape-based similarity for curves or planes. For us, data series represent $y$-coordinate of two-dimensional nozzle shape. $\mathrm{X}$-coordinate is represented by the index of $\mathrm{i}^{\text {th }}$ value. This is valid for consistent datasampling of both, series $c$ and $c^{\prime}$. For non-consistent data sampling we need to define location vector $x$ instead of single coordinate as shown at the figure 3 . 


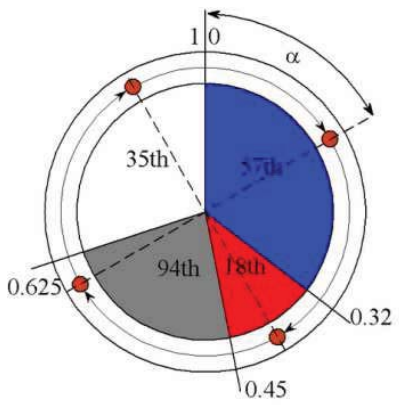

Figure2:Stochastic universal sampling

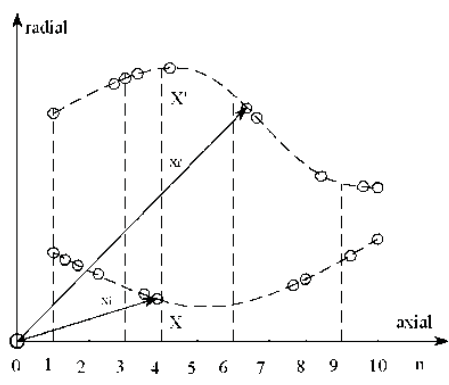

Figure 3: Inconsistent data sampling. Location vectors in $\mathbf{R}^{\mathbf{2}}$.

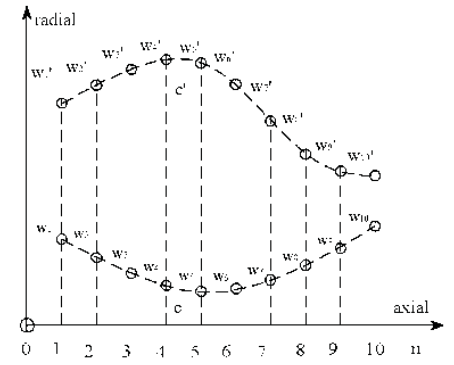

Figure 4: Consistent data sampling.

Equation (4) is an extension for location vectors, where the spatial definition of curve is given by the location vector series. If each location vector $x$ is a vector in space $\mathbf{R}^{\mathbf{2}}$ and vectors series are $X=\left\langle x_{1}, x_{2}, \ldots x_{n}\right\rangle, X^{\prime}=\left\langle x_{1}^{\prime}, x_{2}^{\prime}, \ldots x_{n}^{\prime}\right\rangle$, the distance of vector series is defined as follows

\section{CFD nozzle optimization}

$$
D\left(X, X^{\prime}\right)=\sqrt{D\left(X_{1}, X_{1}^{\prime}\right)^{2}++\cdots+D\left(X_{n}, X_{n}^{\prime}\right)^{2}} .
$$

Optimization of nozzle shown at the fig. 1 passes over two separate procedures. The first part includes selection of location vectors from parametric space and consequential optimization of vector sequence in order to minimize the overall deformation defined with the application of equation (3) as

$$
D_{\text {tot }}=\sum_{i=1}^{i=N} D\left(c_{i}, c_{i+1}\right), \text { where } N=\text { no. of vectors }=243 \text { and } i=1,2, \ldots, N \text {. }
$$

Second part includes CFD simulation of flow within designed nozzle. We used commercial code FLUENT to solve 2D RANS equations by final volume technique. For all equations, convective terms are discretized using a second-order upwind scheme; inviscid fluxes are derived using a second order flux splitting achieving the necessary upwinding and dissipation close to shocks. Diffusion terms are always cast into a central difference form. The discretized system is solved in a coupled way. We used k-omega turbulence model introduced by Wilcox in [12] to complete the system of governing equations. The standard k-omega model has a very strong sensitivity to the free stream conditions. That is why we used the shear stress transport (SST) modification of the k-omega model provided by Menter in [13]. SST overcomes this deficiency because it is based on a blending approach. Indeed, to archive the different desired features, the standard kepsilon model turned into k-omega formulation, is used from the wake region and outside; while the original model is used in the near wall region. To perform these features, a blending function $F_{1}$ is designed to be one inside the boundary layer and to change gradually to zero in the wake region, if present. The resulting equation for $\omega$ is

$$
\frac{\partial}{\partial t}(\rho \omega)+\frac{\partial}{\partial x_{i}}\left(\rho \omega u_{i}\right)=\frac{\partial}{\partial x_{j}}\left[\left(\mu+\frac{\mu_{t}}{\sigma_{\omega}}\right) \frac{\partial \omega}{\partial x_{j}}\right]+G_{\omega}-Y_{\omega}+\left(1-F_{1}\right) D_{\omega}
$$

More detailed view and expressions of all terms in equation 6 is provided by Menter in [13]. The criterion for assessing convergence was based on the root mean square of the density residues expressed by

$$
R(\xi)=\left[\sum_{i=1}^{M}\left(\frac{\partial \xi}{\partial t}\right)_{i}^{2}\right]^{1 / 2}
$$


Where $M$ is the number of grid points and $\xi$ is the variable considered to check (mass, energy, momentum, etc.). Generally, computations are stopped when residuals fall below $1 \times 10^{-6}$ and when the solution is was no longer changing. In addition, at convergence, the mass imbalance is checked on each inlet and outlet boundaries. All mentioned requirements were successfully meet in 400 iteration steps after grid update with Courant-Friedrichs-Lewy (CFL) number set to 50. Boundary conditions were set to pressure inlet/outlet with non-reflecting boundary condition enabled. Turbulent intensity at the nozzle inlet was set to $3 \%$ and gauge total pressure to $p_{01}=0 \mathrm{~Pa}$. Outlet gauge pressure was set to $p_{\mathrm{E}}=-70000 \mathrm{~Pa}$. Symmetric computational domain consists of 65000 quadrilateral elements with original mesh size $0.2 \mathrm{~mm}$. Gradual boundary layer in ten levels from the size of $0.005 \mathrm{~mm}$ in wall adjacent cell to free stream value was modelled to keep the wall $y^{+} \approx 1$. We applied dynamic mesh model update to derive most benefits of minimal deformation path. User Defined Function (UDF) described in [14] and applied by Dvořák in [15] is compiled to drive the wall boundary deformation over whole sequence of nozzle shapes (vectors). The original domain that underlies deformation has simple linear shape as shown at the top of figure 5. UDF updates the $y$-coordinate ( $y_{\text {node }}$ ) of each node at time $t+1$ (new shape) according to spring based deformation defined as follows

$$
y_{\text {node }}^{t+1}=\frac{y_{\text {node }}^{t}}{y_{\text {wall }}^{t}} y_{\text {wall }}^{t+1} \text {. }
$$

There is an example of originate shape deformation, at the figure 5. Flow, turbulence and status variables in control volume and their fluxes are preserved for the newly formed mesh from previous time step $t$. With the minimal mesh deformation, the complete converging process based on equation (7) takes only incomparable small number of steps in compare to "zero" initialization. It took about 27 hours on single CPU to complete the solution of all 243 variants within 100000 iterations.

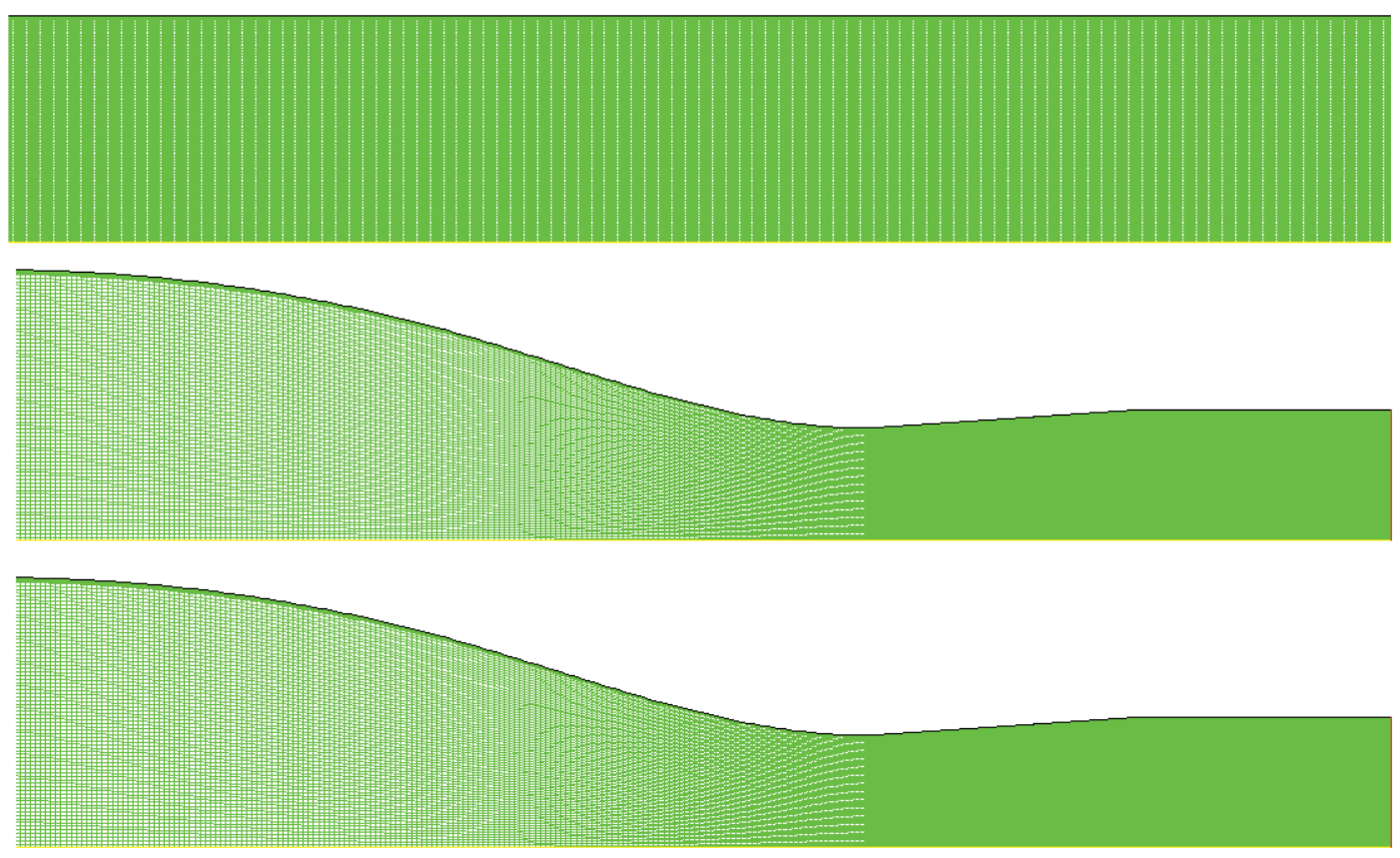

Figure 5: First three shapes from the sequence of deformed domains with mesh. 


\section{RESULTS}

The genetic algorithm took about 4 hours to find best acceptable sequence of all variations from table 1 . The solution progress at the figure 6 shows the best and average deformation (vector sequence) during evolution. Best resulting deformation path gains half of initial value. Although this good improvement, slow abandonment of local optimum found about $3000^{\text {th }}$ generation is obvious. Thus, efficiency of GA should be improved before next use. The influence of mutation and recombination operators is to be studied to increase an efficiency of mentioned GA.

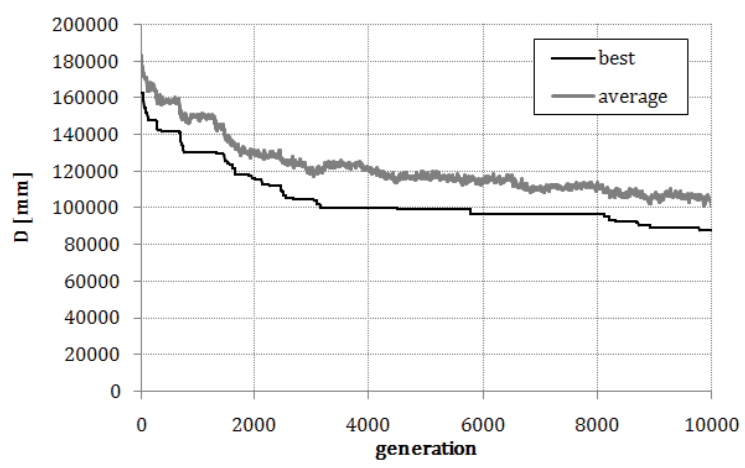

Figure 6: Evolution of minimal and average deformation of nozzles sequence.

Objective of optimization process was established in according to wind tunnel nozzle requirement as

, where $i=1,2, \ldots N, N$ is the no. of cell faces at the exit boundary.

In equation (7), $A_{i}$ is the boundary cell face area and $\beta$ has meaning of momentum coefficient at nozzle exit defined as follows

The value of $\beta$ captures the velocity profile. $\beta$ closer to the one, the better-balanced, shocked-free and boundary layer separation-free velocity profile at nozzle exit realized. The results of computed momentum coefficient for all variants are shown at the figure 7 .
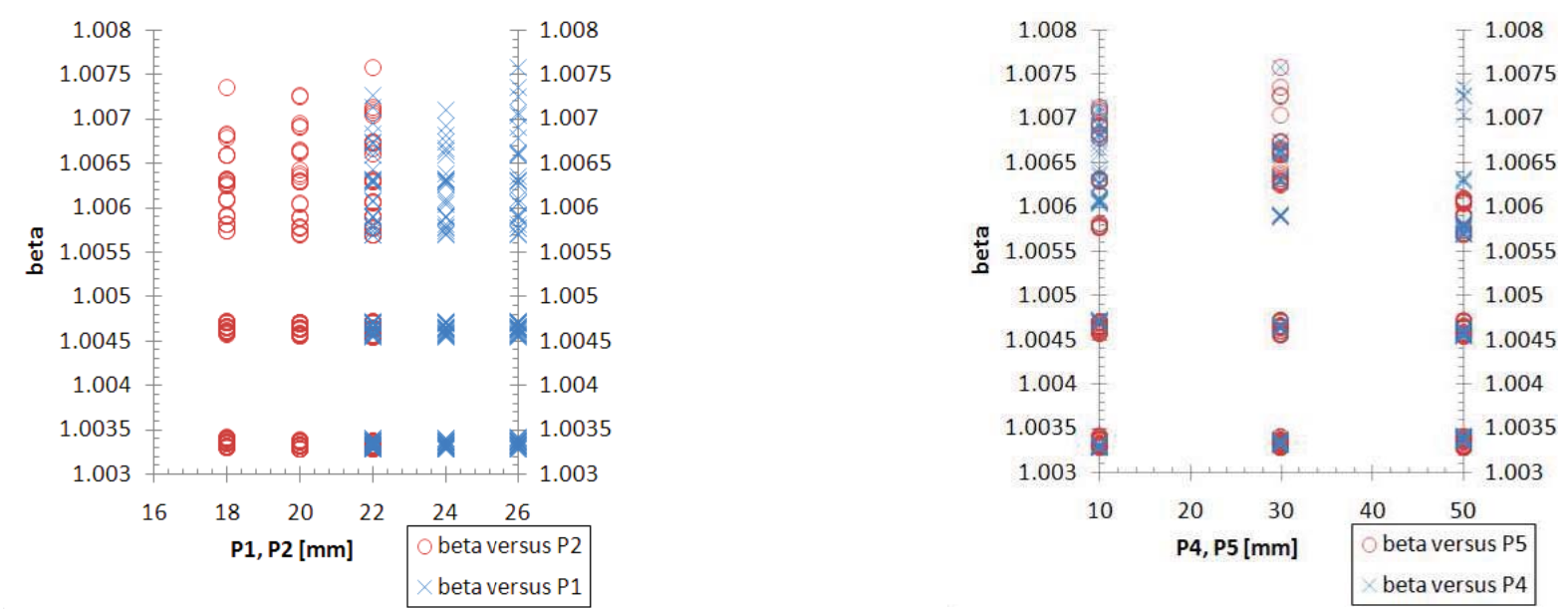

Figure 7: Momentum coefficient at nozzle exit versus P1, P2 (left) and P4, P5 (right). 
It is possible to get exit velocity profile with the same behaviour for all values of P1 and P2 or even P4 and P5 as seen at figure 7. Generally, properties of exit flow are segregated in three separate levels. Two of them located at the lower end of beta within short interval corresponds to $\mathrm{Ma} \approx 1.28$ and $\mathrm{Ma} \approx 1.35$ and third group occupies relatively large interval corresponds to $\mathrm{Ma} \approx 1.42$. The lower level of $\mathrm{Ma}$, the lower level of $\beta$. The influence of radii ratio P5/P4 on exit Mach number supports the conclusion, that the behaviour of velocity profile strongly depends on operating Mach number. The values of $\beta$ are given mainly by the strength of shock attacking nozzle exit as shown at the figure 8 and 9. Although no general condition about parameters was found, the best variant had
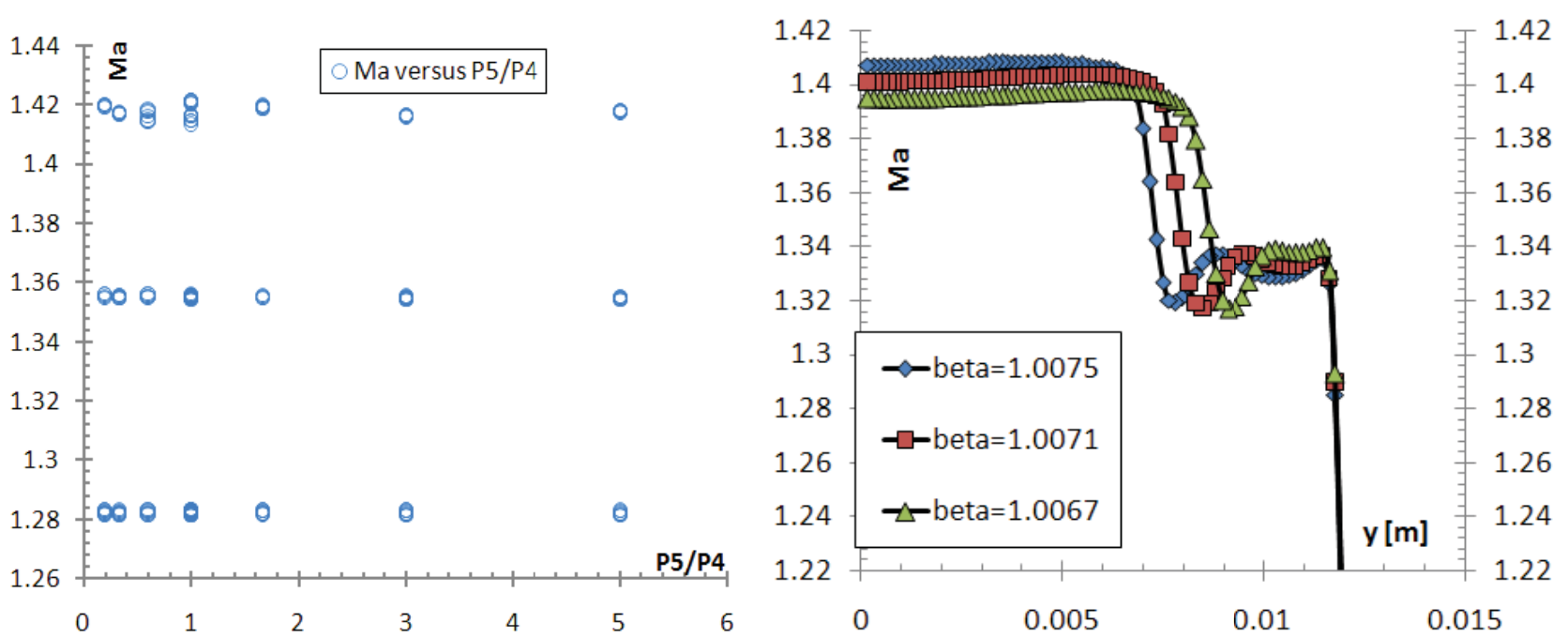

Figure 8: Exit Mach number versus P5/P4.

Figure 9: Selected profiles of Ma at exit.

to be picked out. We decided to choose one with exit $M a=1.42$ and lowest momentum coefficient available. The case no. 225 fits this requirement with parameters $P 1=26 \mathrm{~mm}$, $\mathrm{P} 2=22 \mathrm{~mm}, \mathrm{P} 3=10.8 \mathrm{~mm}, \mathrm{P} 4=50 \mathrm{~mm}, \mathrm{P} 5=50 \mathrm{~mm}$ with $\beta=1.00658$. Other models with varying intake parameters $\mathrm{P} 1$ and $\mathrm{P} 2$ with constant $\mathrm{P} 4, \mathrm{P} 5=50 \mathrm{~mm}$ approximate best solution, too.

\section{Conclusion}

The new optimization procedure was established and discussed. We tested the procedure by the optimization of supersonic wind tunnel primary nozzle. From results, only negligible influence of subsonic nozzle-part on flow pattern downstream is proved. On the other hand, very strong impact of diverging shape and Mach number on velocity profile at the nozzle exit is proved. Consequentially, additional optimization of diverging interval with fixed subsonic part is to be done to explore this part of optimization space more precisely. The best variant available from actual parameterization comes with the highest radii available. This conforms to theoretical hypothesis about interaction of compression waves into shock. We will repeat the optimization with the highest radii, geometrically available, instead of presented $\mathrm{P} 5_{\max }=50 \mathrm{~mm}$, to prevent shock induction.

This work was supported by the Czech Science Foundation, grant no. P101/10/1709 "Nozzles and diffusers in ejectors". 


\section{REFERENCES}

[1] Shapiro, A. H. The dynamics and thermodynamics of compressible fluid flow. New York: The Ronald Press Company, 1953. ISBN 0826080758.

[2] Moeckel, W. E. Interaction of oblique shock waves with regions of variable pressure, entropy and energy. Washington : NACA, 1952. TN 2725.

[3] Kolář, J. and Šafařík, P. Interaction of oblique shock wave with shear layer. Plzeň : In Proceedings of the International Conference XXVII: Meeting of Departments of Fluid Mechanics and Thermomechanics, 2008. pp. 163-170. ISBN 978-80-7043-666-0.

[4] Dvořák, V. and Kolář, J. Shape optimization of supersonic ejectors with several primary nozzles. Lisboa, Portugal : In the 2nd International Conference on Engineering Optimization, 6.-9. september 2010. ISBN 978-989-96264-3-0.

[5] Dvořák, V. Shape optimization of supersonic ejector for supersonic wind tunnel. s.I. : In.: Applied and Computational Mechanics, 2010. pp. 15-24. ISSN 1802-680X.

[6] Breitkopf, P. and Coelho, R. F. Multidisciplinary design optimization in computational mechanics. s.I. : Wiley-ISTE, 2010. ISBN-10 1848211384.

[7] Dvořák, V., Stupka, O. and Kolář, J. Design and numerical calculation of variable test section for small supersonic wind tunnel. Rožnov pod Radhoštěm : In Proceedings of the International Conference XXVIII: Meeting of Departments of Fluid Mechanics and Thermomechanics, 23.-26.6.2010. ISBN 978-80-248-2244-0.

[8] Hynek, J. Genetic algorithms and genetic programing (in Czech). Prague: Grada Publishing, a. s., 2008. ISBN 978-80-247-2695-3.

[9] Holland, J. H. Adaption in natural and artificial systems. Ann Arbor, Michigan : The University of Michigan Press, 1975.

[10]. Zelinka, I. et al. Evolutionary computational techniques (in Czech). Prague : BENtechnická literatura, 2008. ISBN 978-80-7300-218-3.

[11] Yanagisawa, Y., Akahani, J. and Satoh, T. Shape-based similarity query for trajectories of mobile objects. Taipei : Mobile Data Management p. 63-77, 2003. ISSN 0302-9743.

[12] Wilcox, D. Reassessment of the scale determining equation for advanced turbulent models. 1988. AIAA J. 26 (11),1299-1310.

[13] Menter, F. R. Two-equations Eddy-viscosity turbulence models for engineering applications. 1994. AIAA J. 32(8), 1598-1605.

[14] Inc., Fluent. Fluent user documentation. Lebanon : s.n., 2006.

[15] Dvořák, V. Optimized Axi-symmetric ejector - experimental and numerical investigation. Liberec: In Experimental Fluid Mechanics,25. - 27. November. pp 34 43., 2009. ISBN 978-80-7372-538-9. 